\title{
The future of medical thoracoscopy in the Kingdom of Saudi Arabia
}

Enas M. Batubara, MD, SBIM. Abmed S. Ibrahim, MD, MSc, Suliman M. Alamro, MD, ABIM.

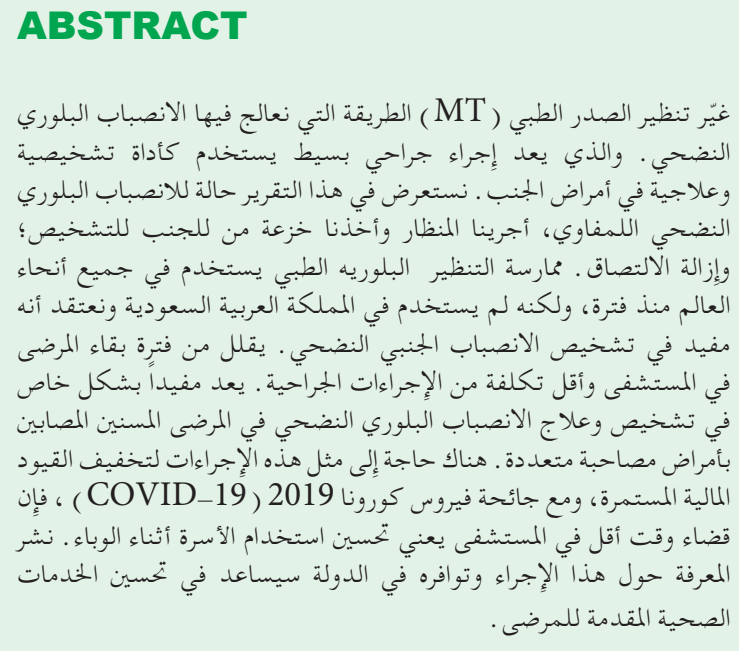

Medical thoracoscopy (MT) has changed how we manage exudative pleural effusion. It is a minimally invasive procedure used as a diagnostic and therapeutic tool in pleural disease. Here, we report a case of a lymphocytic exudative pleural effusion that needed a pleural biopsy for diagnosis. Medical thoracoscopy was performed, a biopsy was taken, and adhesiolysis was performed. Medical thoracoscopy has been practiced for a while worldwide, but it has not been utilized in the Kingdom of Saudi Arabia and as we believe that it is useful in diagnosing exudative pleural effusions. It limits patients in hospital-stay and it may be less costly than surgical procedures. It is especially helpful in diagnosing and treating pleural effusions in elderly patients with multiple comorbidities. Such procedures are needed to ease ongoing financial constraints, and with the 2019 coronavirus disease (COVID-19) pandemic, less time in the hospital means better utilization of beds during the pandemic. Spreading the knowledge about this procedure and its availability in the country will improve the health services provided to the patients.

Keywords: medical thoracoscopy case report, pleural biopsy case report, pleural effusion case report
Saudi Med J 2021; Vol. 42 (8): 903-907

doi: 10.15537/smj.2021.42.8.20200716

From the Department of Medicine, Pulmonary Division, Prince Sultan Military Medical City, Riyadh, Kingdom of Saudi Arabia.

Received 14th February 2021. Accepted 7th June 2021.

Address correspondence and reprint request to: Dr. Enas $M$. Batubara, Department of Medicine, Pulmonary Division, Prince Sultan Military Medical City, Riyadh, Kingdom of Saudi Arabia. E-mail:ebatubara@gmail.com

ORCID ID: https://orcid.org/0000-0003-3076-7854

Ex xudative pleural effusions are common clinical problems encountered by many physicians. ${ }^{1}$ Pleural fluid analysis is often non-diagnostic, and further workup is required to identify the underlying etiology. ${ }^{2}$ Exudative effusions are frequently related to underlying malignancy or infections such as tuberculosis (TB), both of which require a timely diagnosis to institute therapy. ${ }^{2}$ However, following thoracentesis and establishing the exudative nature of a pleural effusion, there is a debate on what is the next optimal step in the diagnosis. ${ }^{3}$

Closed pleural biopsy, video-assisted thoracoscopic surgery (VATS), computed tomography (CT) guided biopsy, and medical thoracoscopy (MT) may be used for pleural biopsy, and each procedure has its advantages and disadvantages. ${ }^{3}$

Medical thoracoscopy is a minimally invasive, single-port endoscopic technique using rigid or semi-rigid thoracoscopes that offers direct visualization of pleural surfaces and channels to perform diagnostic and therapeutic procedures. Patients typically receive moderate sedation and breathe spontaneously throughout the procedure. This allows for targeted parietal pleural biopsies and various therapeutic interventions, including adhesiolysis, pleurodesis, and concurrent tunneled pleural catheter placement. ${ }^{4}$

We present a patient with an exudative pleural effusion managed by MT with pleural biopsies and lysis of adhesions.

Case Report. A 24-year-old male with a 20 pack-year history of smoking presented to the emergency 
room with a history of fever and shortness of breath of 2 weeks duration. He sought medical advice and received antibiotics for 7 days with no improvement in his symptoms. He reported a history of weight loss and loss of appetite for the last 4 months, and occasional use of alprazolam as a recreational drug. There were no symptoms suggestive of auto-immune disease.

On examination, he looked cachectic and apart from a fever of $38.5^{\circ} \mathrm{C}$, all his vitals were within normal. Chest examination revealed dullness on percussion, decreased breath sounds at the left lower chest, and no other significant signs. The chest $\mathrm{x}$-ray showed left-sided moderate pleural effusion. The bedside ultrasound showed a moderate amount of pleural effusion, freely moving fibrin bands and no obvious loculation. A chest drain was inserted yielding turbid yellow fluid. Pleural fluid tests and serum lab results are given in Table 1.

Computed tomography with contrast was carried out (Figure 1) as part of the investigation. The patient's symptoms persisted and chest drain continued draining. The preliminary diagnosis of the patient's case was lymphocytic exudative pleural effusion, with a history of night sweats, loss of appetite and thickened enhanced pleura in the CT chest. At this stage, a pleural biopsy was recommended. Differential diagnosis of such a case includes pleural TB, malignant pleural effusion, auto-immune disease, and nonspecific pleuritis (less likely).

Medical thoracoscopy is the least invasive with the highest yield in this case, as CT guided biopsies were deferred by the radiologist due to the absence of pleural nodules and lesions that could be targeted. Closed pleural biopsy was felt to have a lower diagnostic yield than MT since the initial mycobacterium TB PCR was negative and malignancy was still a possible cause of this patient's pleural effusion given his significant smoking history. There were no significant loculations on $\mathrm{CT}$ (Figure 1).

The procedure was explained to the patient, including the need for local anesthesia with moderate sedation. The patient agreed and provided a signed consent. This procedure is usually performed in the bronchoscopy suite where interventional pulmonologists administer the sedatives plus opioid assisted by another physician, a nurse and a technician. The decision was taken to perform the case in the operation room with the

Disclosure. Authors have no conflict of interests, and the work was not supported or funded by any drug company.
Table 1 - Pleural fluid and serum test findings.

\begin{tabular}{lc}
\hline Laboratory test & Results \\
\hline Pleural PH & 7.38 \\
Pleural total protein & $50 \mathrm{~g} / \mathrm{L}$ \\
Pleural lactate dehydrogenase & $431 \mathrm{U} / \mathrm{L}$ \\
Pleural amylase & $51 \mathrm{U} / \mathrm{L}$ \\
Pleural cell predominance & Lymphocyte $90 \%$, polymorph $10 \%$ \\
PCR-mycobacterium TB in & Negative \\
pleural fluid & Negative \\
Pleural fluid ziehl-neelsoen stain/ & 137 \\
acid fast stain & $104 \mathrm{mg} / \mathrm{l}$ \\
Lactate dehydrogenase & 6.5 \\
CRP & Negative \\
WBC & $0.06 \mathrm{ng} / \mathrm{ml}$ \\
Sputum AFB & \\
Procalcitonin & AFB: acid-fast bacilli \\
\multicolumn{2}{c}{ TB: tuberculosis, CRP: c-reactive protein, WBC: white blood cells, } \\
\multicolumn{2}{c}{}
\end{tabular}

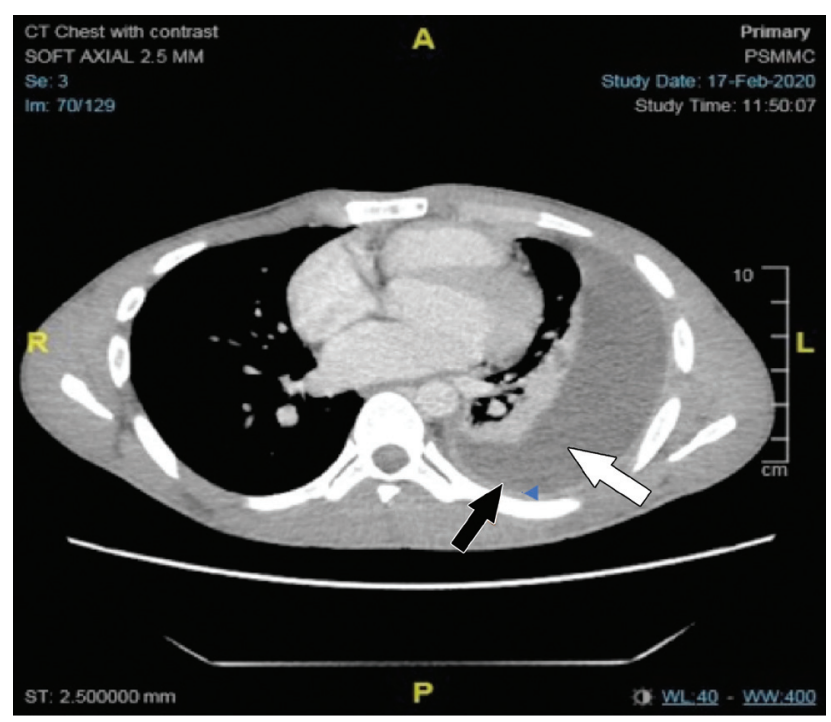

Figure 1 - Computerized tomography with contrast. Pleural fluid (white arrow) and thickened pleura (black arrow).

anesthetist's support to provide moderate sedation so that other team members could fully focus on the procedural technique.

Procedural steps

1. Radiological review

2. Positioning the patient. The patient lied down on the right side (the healthy side) in a lateral decubitus position with left side upward. His arm was raised over the head with the help of a sling. A pillow was placed on the table under the patient's flank, causing the spine to flex laterally. An axillary roll was used to protect the brachial plexus. 
3. Marking the point of entry. A bedside ultrasound was carried out and the pleural space measured, which was approximately $7 \mathrm{~cm}$ from the chest wall to the visceral pleural. There were few fibrin bands that were moving freely. The point of entry was marked, which was in the mid axillary line in the 5 th intercostal space on the upper border of the rib.

4. The area was sterilized and draped

5. Time-out

6. Local anesthesia and sedation. Two percent of lidocaine was used to anesthetize the skin and the pleura at the entry site. He received $2 \mathrm{mg}$ of Midazolam 10 minutes before the procedure. He was given dexmedetomidine $(0.5 \mathrm{mcg} / \mathrm{kg})$ slow intravenous infusion over $10 \mathrm{~min}$ and propofol $(50$ $\mathrm{mcg} / \mathrm{kg} / \mathrm{min}$ ) infusion. The patient further received $200 \mathrm{mg}$ of propofol over $90 \mathrm{~min}$ and $25 \mathrm{mcg}$ of dexmedetomidine over the same period.

7. Monitoring of the patient. Oxygen saturation, end tidal $\mathrm{CO}^{2}$, blood pressure, and level of conciseness were monitored continuously. He was kept on oxygen $(3 \mathrm{~L} / \mathrm{m})$ through nasal cannula.

8. Access to the pleural space. A $12-\mathrm{mm}$ incision and dissection were done until the pleural space was accessed. An $8 \mathrm{~mm}$ disposable trocar was inserted, whereafter an Olympus semi-rigid pleuravideoscope LTF-260 was inserted through the trocar, and around $850 \mathrm{ml}$ of turbid (free-flowing fluid) was aspirated.

9. Examination and biopsies. An examination of the chest wall showed an inflamed and hyperemic visceral and parietal pleura, covered with a "fibrinous patch". The visceral pleura appeared to be covered with fibrin. (Figure 2). The few adhesions were dissected using the scope and the forceps (Figure 3), and approximately 12 biopsies were taken from the parietal pleura; six were sent in saline and another six in the formalin. There was no bleeding, and the patient was stable.

10. Chest drain insertion. A $24 \mathrm{~F}$ chest drain was inserted.

Post procedure. The patient was kept on a chest drain connected to suction, and around $400 \mathrm{ml}$ was drained over a 48-h period. As his lung was not expanding and no evidence of an air leak, thoracic surgeons were consulted. It was decided to remove the chest drain and follow up the patient after anti-Tb for possible decortication if his lung did not expand.

Results and patient's treatment course. His pleural biopsy results were: 1) histopathology: caseating granuloma; and 2) PCR for mycobacterium TB was positive. Case timeline shown in Table 2.

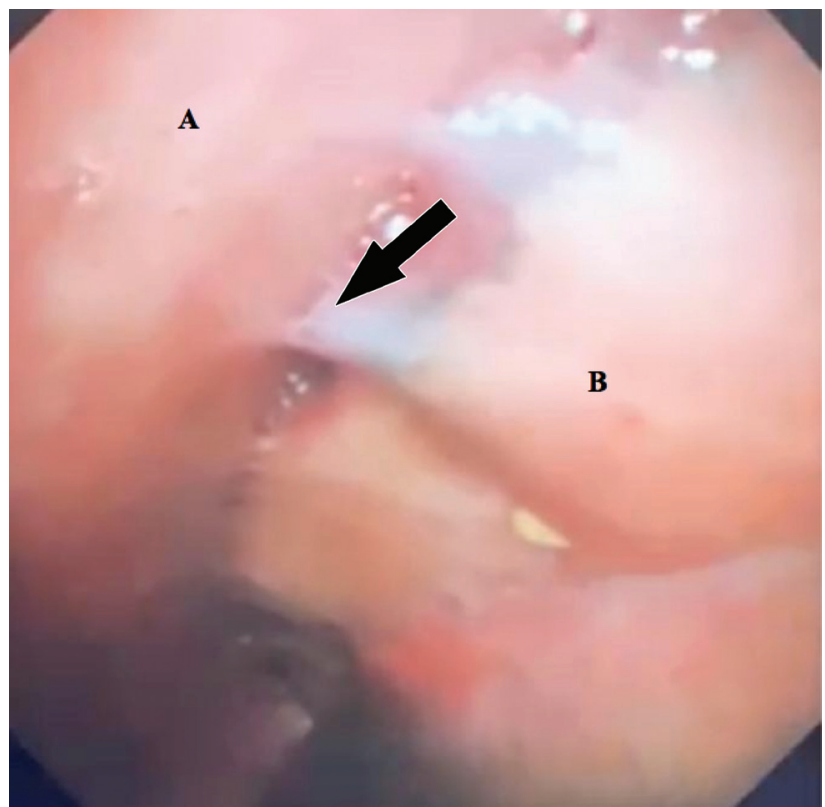

Figure 2 - Thoracoscopic image of the lung and chest wall. A) Chest wall and B) collapsed lung covered with fibrin. Fibrin attaching the lung to the chest wall (arrow).

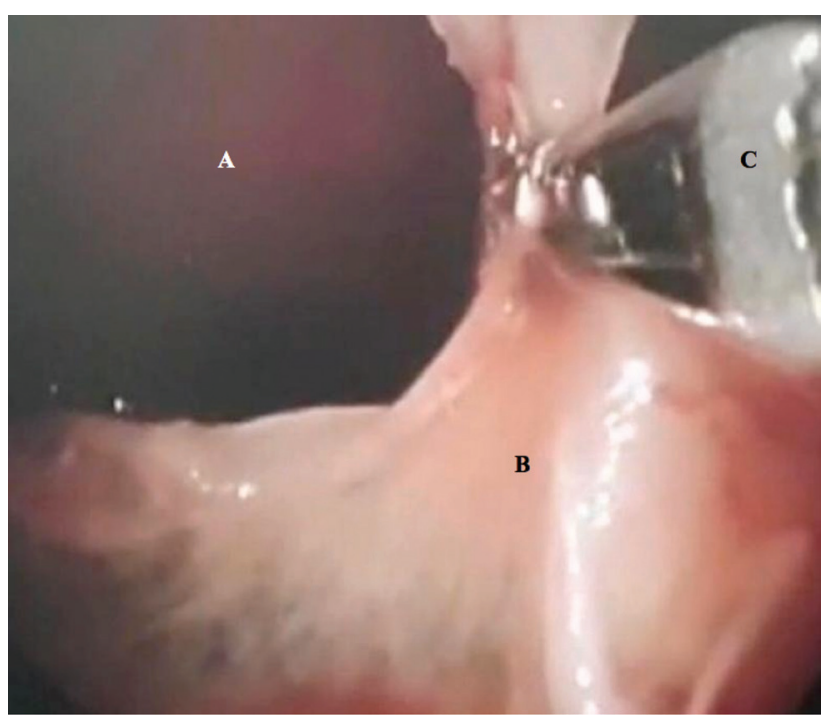

Figure 3 - Thoracoscopic view of the fibrin bands "locules' being dissected. A) Chest wall, B) fibrin, and C) forceps.

Follow-up and follow up. The patient was started on an anti-TB (Isoniazid $300 \mathrm{mg}$, Rifampicin $600 \mathrm{mg}$ orally, Ethambutol 1200 mg, Pyrazinamide 1500 mg and pyridoxine $40 \mathrm{mg}$ ) once daily. He tolerated his medication with no side effects. The patient was given an appointment to be seen after 2 weeks in the clinic. During his follow-up, he gained weight, his shortness of breath improved markedly, and his lung re-expanded. 
Table 2 - Case timeline.

\begin{tabular}{|c|c|c|c|}
\hline \multirow{3}{*}{$\begin{array}{l}\text { Dates } \\
09 \text { February } 2020\end{array}$} & \multicolumn{3}{|c|}{ Relevant past medical history and interventions } \\
\hline & \multicolumn{2}{|c|}{ 24-year-old male smoker (20 Pk/year) } & \multirow[b]{2}{*}{ Interventions } \\
\hline & Summaries from initial and follow-up visits & Diagnostic testing & \\
\hline 16 February 2020 & $\begin{array}{l}\text { Complains of chest pain on right side. } \\
\text { Pleural fluid analysis showed lymphocytic } \\
\text { pleural effusion. }\end{array}$ & $\begin{array}{l}\text { CT of the chest was carried out which showed } \\
\text { large loculated left-sided pleural effusion with thick } \\
\text { enhancing pleura. Thoracoscopy was carried out and } \\
\text { pleural biopsy was taken which showed necrotizing } \\
\text { granuloma. PCR TB tested on } 21 \text { February } 2020 \\
\text { displayed negative results. }\end{array}$ & $\begin{array}{l}\text { Anti-TB was started on } \\
24 \text { February } 2020 \text { in } \\
\text { the form of rifampicin, } \\
\text { isoniazid, pyrazinamide, and } \\
\text { ethambutol. }\end{array}$ \\
\hline 16 March 2020 & $\begin{array}{c}\text { Anti-TB medication was started on } 24 \\
\text { February } 2020 .\end{array}$ & $\begin{array}{l}\text { TB culture carried out on } 16 \text { March } 2020 \text { tested } \\
\text { positive }\end{array}$ & \\
\hline 22 December 2020 & $\begin{array}{l}\text { Patient had no physical follow-up since } \\
\text { March due to COVID } 19 \text { pandemic. No } \\
\text { complains of chest pain, no cough, fever } \\
\text { sputum. Weight gain of } 5 \mathrm{~kg} \text {. looks healthy. }\end{array}$ & $\begin{array}{l}\text { Chest X-ray shows resolution of left-sided pleural } \\
\text { effusionx residual pleural thickening and mild volume } \\
\text { loss, no new infiltrates bilaterally. }\end{array}$ & $\begin{array}{l}\text { Stopped treatment himself in } \\
\text { August } 2020 \\
\text { (completed } 6 \text { months of Anti } \\
\text { TB medication). }\end{array}$ \\
\hline
\end{tabular}

CT: computerized tomography, PCR: polymerase chain reaction, TB: tuberculosis

Discussion. Twenty percent of exudative pleural effusions remain undiagnosed after pleural fluid analysis, and need further investigation. ${ }^{2}$ In patients with lymphocytic exudative pleural effusions, the differential diagnosis should include pleural TB, malignancy, lymphoma, and auto-immune disease. The patient had no clinical symptoms or labs consistent with an auto-immune disease. Given the effusion was exudative with 90\% lymphocytes, and high protein, CT Chest showed no pleural nodules or masses and sputum AFB and pleural fluid TB PCR were negative, there was still a concern about tuberculosis. Pleural biopsy is the gold standard for diagnosing pleural TB $5 .{ }^{5}$

A closed pleural biopsy versus MT was contemplated, as closed pleural biopsy has been useful since it was first used 50 years ago. It is accessible, minimally invasive, and cost-effective. The diagnostic yield for malignancy may be less than $60 \%,{ }^{6}$ while the diagnosis of TB is $80-87 \%$ provided at least 6 specimens are obtained. ${ }^{7}$ Medical thoracoscopy is also minimally invasive yet outperforms closed pleural biopsy because it enables direct visualization of the biopsy target. It has a higher diagnostic yield (91-95\%) for pleural malignancy and up to a $100 \%$ yield for pleural TB. ${ }^{7}$ Furthermore, it allows for therapeutic interventions including chemical pleurodesis and adhesiolysis. It is distinguished from surgical thoracoscopy by typically using only one port of entry and spontaneous ventilation. Patients may be discharged on the same day of the procedure. Unlike surgical thoracoscopy, it is less invasive and needs one port of entry without one-lung ventilation and no general anesthesia.

In an era where the trend is for minimally invasive procedures and short in-hospital stays, MT has become a preferred option in many parts of the world. Medical thoracoscopy has been used for a while by interventional pulmonologists, Although, MT was only recently used for treating pleural infection by adhesiolysis in loculated pleural effusion. In such cases, MT is considered as a drainage procedure mid-way between drainage by chest drain and video assisted thoracoscopic surgery/ thoracotomy.

Medical thoracoscopy is a new procedure in this region and reporting it will hopefully spread the knowledge about its availability and utility.

In conclusion, MT can be carried out in an available setting, and the presence of anesthetist's is helpful as this decreases the procedure time and lowers the risks.

Acknowledgment. The we gratefully extend our appreciation to Dr. Alaa Y. Sabbagh for reviewing the patient's CT. We also acknowledge American Manuscript Editors for English language Editing.

\section{References}

1. Anevlavis S, Varga C, Nam TH, Man RWC, Demetriou A, Jain $\mathrm{N}$, et al. Is there any role for thoracoscopy in the diagnosis of benign pleural effusions. Clin Respir J 2019; 13: 73-81.

2. Nugent K, Berdine G, Test V. What is the next step in a patient with an undiagnosed exudative pleural effusion? Am J Med Sci 2020; 360: 211-212.

3. Lee P, Folch E. Thoracoscopy: advances and increasing role for interventional pulmonologists. Semin Respir Crit Care Med 2018; 39: 693-703.

4. Kyskan R, Li P, Mulpuru S, Souza C, Amjadi K. Safety and performance characteristics of outpatient medical thoracoscopy and indwelling pleural catheter insertion for evaluation and diagnosis of pleural disease at a tertiary center in Canada. Can Respir J 2017; 2017: 9345324.

5. Antonangelo L, Faria CS, Sales RK. Tuberculous pleural effusion: diagnosis \& management. Expert Rev Respir Med 2019; 13: 747-759. 
6. Metintas M, Yildirim H, Kaya T, Ak G, Dundar E, Ozkan R, et al. CT scan-guided Abrams' needle pleural biopsy versus ultrasound-assisted cutting needle pleural biopsy for diagnosis in patients with pleural effusion: a randomized, controlled trial. Respiration 2016; 91: 156-163.
7. Casalini AG, Mori PA, Majori M, Anghinolfi M, Silini EM, Gnetti L, et al. Pleural tuberculosis: medical thoracoscopy greatly increases the diagnostic accuracy. ERJ Open Res 2018; 4: 00046-02017. 